\title{
Dynamic changes in PD-1 and PD-L1 expressions in cases with Hodgkin Lymphoma
}

\author{
Semra Paydas ${ }^{1 *}$, Emine Kilic Bagir ${ }^{2}$ and Melek Ergin ${ }^{2}$ \\ ${ }^{1}$ Faculty of Medicine, Departments of Medical Oncology, Cukurova University, Oncology Adana/Turkey \\ ${ }^{2}$ Faculty of Medicine, Departments Pathology, Cukurova University, Oncology Adana/Turkey
}

\begin{abstract}
Objective: The aim of this study is to detect the PD-1 and PD-L1 expressions before and after treatment in primary refractory (PR HL) and relapsed/refractory Hodgkin Lymphoma (RR HL). PD-1 and PD-L1 expressions were evaluated in 17 cases with RR HL by immunohistochemistry.

Method: Immunohistochemistry was used to detect PD-1 and PD-L1 expressions. Tumor cells and peritumoral microenvironment were evaluated separately. More than 5\% expression in microenvironment and/or Reed Sternberg accepted as positive.

Results: PD-1 expression in in microenvironment and RS cells in 4 and 8 cases and 0 and 4 cases in first diagnosis and after treatment, respectively. PD-L1 expression in diagnosis was detected in 3 cases in microenvironment and in 9 cases after treatment. PD-L1 expression at the first diagnosis in RS cells was detected in 2 cases and after treatment in 5 cases.
\end{abstract}

Conclusion: PD-1 and/or PD-L1 expressions after treatment are seen in the majority of cases. Changes in PD-1/PD-L1 expression is frequent in cases with PR and RR HL, and the detection of PD-1/PD-L1 expression in samples taken during RR disease may be important for immunotherapy.

\section{Introduction}

Immunotherapies targeting PD-1 and PD-L1 present promising treatment options for many malignant diseases. Blockade of this pathway named checkpoint blockade therapy (CBT) in solid tumors especially in melanoma showed practice changing activity [1-4]. In contrast to solid tumors, field of CBT in hemopoietic neoplasias is still in infancy but more promising results willbe seen in near future [5-7]. However Hodgkin lymphoma (HL) is privileged neoplasia for this strategy. Because PD-L1 and PD-L2 are key targets for 9p24.1 amplification which is recurrent genetic abnormality in HL. 9p24.1 amplification includes Janus kinase 2 and gene-dosagedependent Janus kinase 2 / signal transducer and activator of transcription activity induces PD-1 ligand transcription [8-10]. EBV infection is common in HL, and is another mechanism of PD-1 overexpression [10]. As a result of these 2 mechanisms, a large proportion of classical HL (cHL) tumors have increased surface expression of PD-L1. PD-1/PD-L1 expression in $\mathrm{HL}$ and their prognostic importance is highly variable [8,9,11-13]. It is very well known that tumors may evade the immune system by upregulation not only of PD-L1 on the tumor cell but also of PD-L1 in its microenvironment. Infections, which are not rare in cases with HL, can induce PD-L1 expression on macrophages. Similarly, the expression of PD-L1 within a tumor and its microenvironment may change with treatment including anti-neoplastic drugs and also radiation [14]. These findings strongly suggest that HL may have a genetic dependence on the PD-1 pathway for survival and that targeting this pathway is highly effective strategy in these cases. For this reason we wanted to explore the PD-1/PD-L1 expressions in relapsed/ refractory cases with HL before and after anti-neoplastic treatment.

\section{Patients And Methods}

PD-1 and PD-L1 expressions were evaluated in seventeen cases with primary refractory $(\mathrm{PR}) \mathrm{HL}$ and relapsed refractory $(\mathrm{R} / \mathrm{R}) \mathrm{HL}$. Female/male ratio was 9/8, age range was between 19 and 59. Formalinfixed, paraffin-embedded tissue samples from 17 cases with $\mathrm{R} / \mathrm{R}$ cases were analyzed in this study. Immunohistochemical (IHC) assay was used to detect PD-1 and PD-L1 expressions.

IHC staining was performed on $5 \mu \mathrm{m}$ sections from tissue using Ab to PD-1 (NAT105/MRQ-22, Ventana, CA, USA) and PD-L1 (Mo Ab CD274/PDL1 AM26531 AF-N.Acris Germany). The visualization system used was the BenchMark XT (Ventana) with heat-induced epitope retrieval ( $\mathrm{CC} 1$ solution Ventana) and the iWiew DAB detection kit (Ventana). The visualization system used was the benchmark XT with enzymatic digestion (ISH Protease 2, Ventana) and the iWiew $_{\text {Blue }}$ detection kit (Ventana). Cases stained with anti-PD-1 and PD-L1 were scored according to intensity of cytoplasmic and/or membranous positivity as follows: 0 (no staining), $1+$ (weak or equivocal staining), $2+$ (moderate staining), or $3+$ (strong staining). Tumor cells and peritumoral microenvironment were evaluated separately. More than

Correspondence to: Semra Paydas, Faculty of Medicine, Departments of Medical Oncology, Cukurova University, Oncology Adana/Turkey, E-mail: sepay@cu.edu.tr

Key words: Hodgkin lymphoma, PD-1, PD-L1, refractory/relapsed disease, immunotherapy

Received: December 18, 2017; Accepted: January 20, 2018; Published: January 23, 2018 
$5 \%$ expression in microenvironment (ME) and/or Reed Sternberg accepted as positive.

\section{Results}

Seventeen cases with HL were evaluated for PD-1 and PD-L1 expressions in biopsy samples at first diagnosis and at samples taken when they relapsed or resistant disease. All cases had cHL; 12 had nodular sclerosing subtype, 4 had mixed cellular type and subtype could not be determined in one case. Eight cases had PR HL and 9 cases had R/R HL. All of the cases had been treated with ABVD regimen as the first line treatment.

Cisplatinum containing DHAP regimen was used for second line or salvage therapy in cases in relapse or refractory disease. Gemcitabine containing regimen was given in third line setting.

Bendamustine, brentuximab vedotin, lenalidomide were given in further lines in some of them.

PR HL cases: Eight cases had PR disease. Before and after chemotherapy PD-1 expression in ME was detected in 2 cases and 4 cases, and in RS cells in none and 2 cases, respectively. Before and after chemotherapy PD-L1 expression in ME was detected in none and 2 cases, and in RS cells in none of the cases, respectively.

R/R HL cases: Nine cases had R/R disease. Before and after chemotherapy, PD-1 expression in ME was detected in none and 2 cases, and PD- 1 expression in RS cells in none and 2 cases, respectively. Before and after chemotherapy, PD-L1 expression in ME was detected in 3 and 7 cases, and in RS cells in 2 and 5 cases, respectively.

When we evaluated all cases, we found that PD-1 expression in ME in 4 cases at the first diagnosis and in 8 cases after treatment. PD- 1 expression in RS cells was not detected at the first diagnosis in none of the cases while in 4 cases after treatment. PD-L1 expression at the first diagnosis was detected in 3 cases in $\mathrm{ME}$ and in 9 cases after treatment. PD-L1 expression at the first diagnosis in ME was detected in 3 cases and in 9 cases after treatment. PD-L1 expression at the first diagnosis in RS cells was detected in 2 cases and after treatment in 5 cases. Table 1 shows the PD- 1 and PD-L1 expressions in PR HL and R/R HL cases before and after anti-neoplastic treatment. PD-1 and PD-L1 expression changes in 2 cases in first diagnosis and relapse have been shown in Figures $1 \mathrm{a}$ and $1 \mathrm{~b}$ and Figures $2 \mathrm{a}$ and $2 \mathrm{~b}$, respectively.

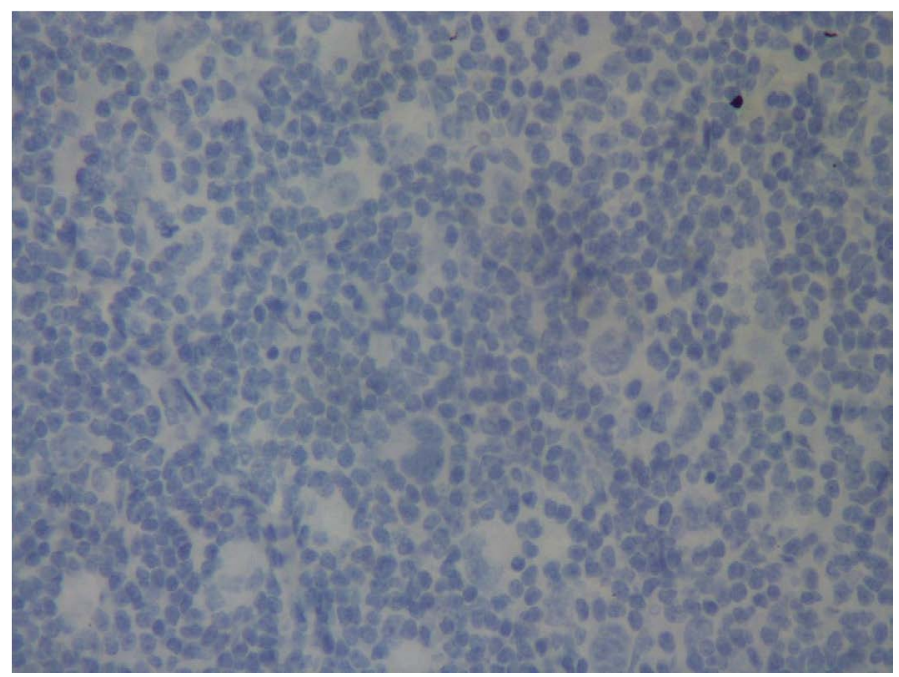

Figure 1a. No PD-1 expression at first diagnosis (Case 17).

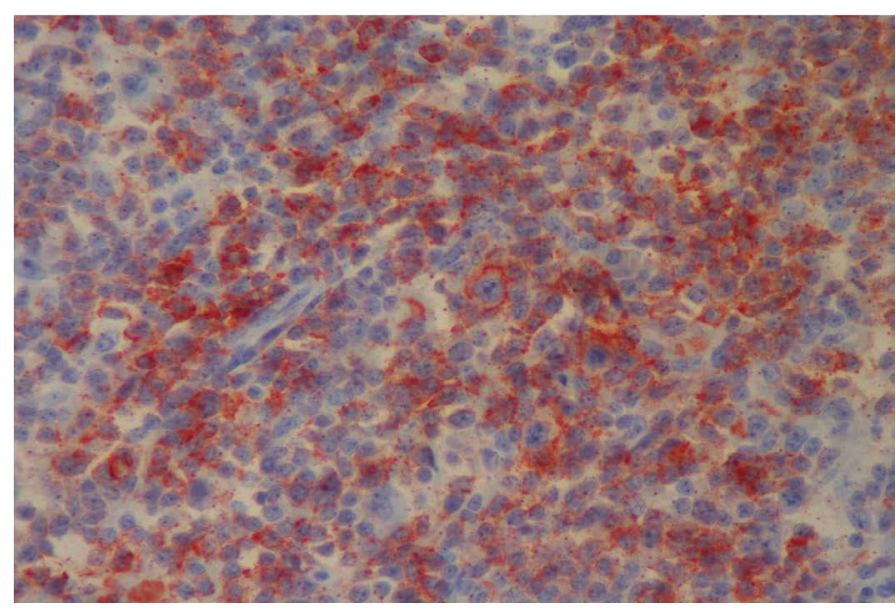

Figure 1b. PD-1 expression at relapse (Case 17).

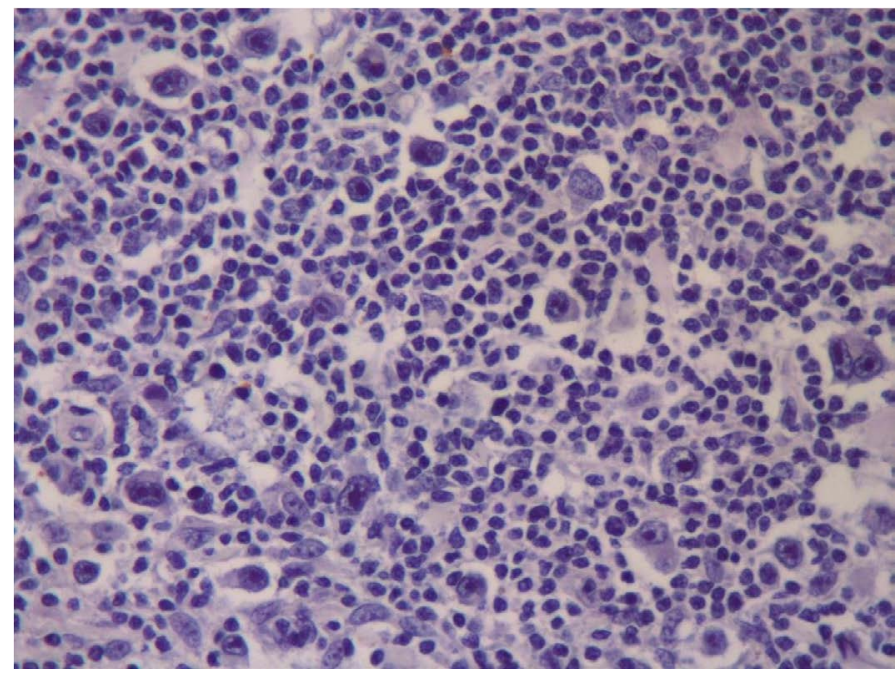

Figure 2a. No PD-L1 expression at first diagnosis (Case 13).

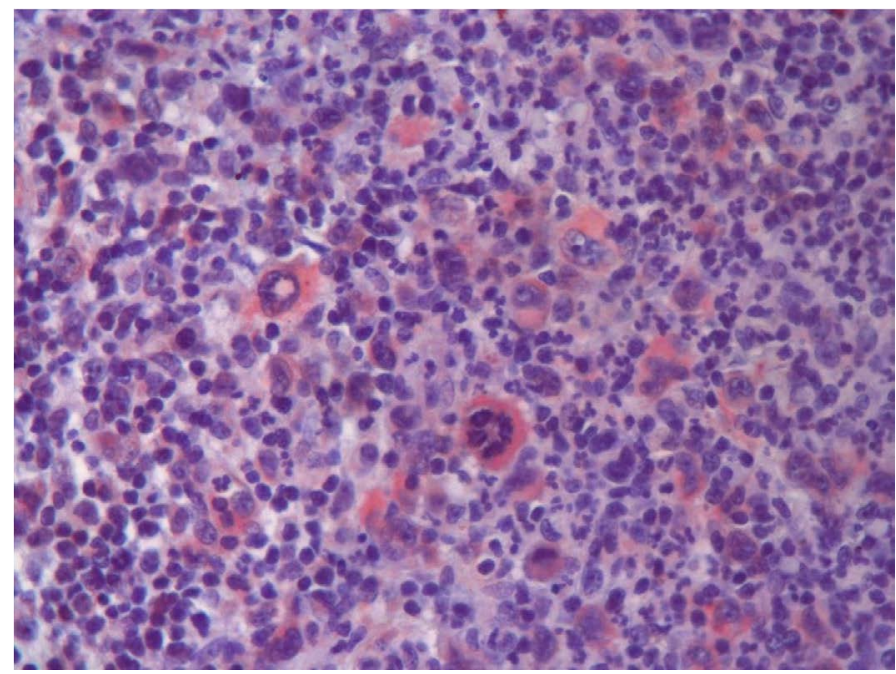

Figure 2b. PD-L1 expression at relapse (Case 13).

\section{Discussion}

In the last years very encouraging results have been shown with immunotherapy in cancer, especially with checkpoint inhibitors. 
Table 1. PD-1 and PD-L1 expressions in first admission and follow up of the patients. NS: nodular sclerosing, MC: mixed cellular, ND: not determined, PR: primary refractory, R/R: relapse /refarctory, ME: microenvironment RS: Reed Sternberg.

\begin{tabular}{|c|c|c|c|c|c|c|c|c|c|c|c|}
\hline \multirow{3}{*}{$\begin{array}{l}\text { Patient } \\
\text { number }\end{array}$} & \multirow{3}{*}{$\begin{array}{l}\text { Sex- } \\
\text { Age }\end{array}$} & \multirow{3}{*}{ Subgroup } & \multirow{3}{*}{$\begin{array}{c}\text { Disease } \\
\text { status }\end{array}$} & \multirow{2}{*}{\multicolumn{2}{|c|}{$\begin{array}{c}\text { Before Treatment } \\
\text { PD-1 }\end{array}$}} & \multirow{2}{*}{\multicolumn{2}{|c|}{$\begin{array}{c}\text { After Treatment } \\
\text { PD-1 }\end{array}$}} & \multirow{2}{*}{\multicolumn{2}{|c|}{$\begin{array}{c}\text { Before Treatment } \\
\text { PD-L1 }\end{array}$}} & \multirow{2}{*}{\multicolumn{2}{|c|}{$\begin{array}{c}\text { After Treatment } \\
\text { PD-L1 }\end{array}$}} \\
\hline & & & & & & & & & & & \\
\hline & & & & ME & RS & ME & RS & ME & RS & ME & RS \\
\hline 1 & Female-23 & NS & PR & + & - & + & - & - & - & + & - \\
\hline 2 & Female-30 & NS & PR & - & - & + & + & - & - & - & - \\
\hline 3 & Male-59 & NS & PR & - & - & - & - & - & - & - & - \\
\hline 4 & Female-21 & ND & PR & - & - & - & - & - & - & - & - \\
\hline 5 & Female-20 & NS & PR & - & - & - & - & - & - & - & - \\
\hline 6 & Male-26 & NS & PR & - & - & + & - & - & - & - & - \\
\hline 7 & Female-27 & NS & PR & - & - & - & - & - & - & - & - \\
\hline 8 & Female-22 & MC & PR & + & - & + & + & - & - & + & - \\
\hline 9 & Male-27 & NS & $\mathrm{R} / \mathrm{R}$ & + & - & + & - & + & + & + & + \\
\hline 10 & Male-30 & $\mathrm{MC}$ & $\mathrm{R} / \mathrm{R}$ & - & - & - & - & - & - & + & + \\
\hline 11 & Female-20 & NS & $\mathrm{R} / \mathrm{R}$ & + & - & + & - & + & - & + & - \\
\hline 12 & Male-58 & NS & $\mathrm{R} / \mathrm{R}$ & - & - & - & - & - & - & + & - \\
\hline 13 & Female-19 & NS & $\mathrm{R} / \mathrm{R}$ & - & - & + & + & - & - & + & + \\
\hline 14 & Male-28 & $\mathrm{MC}$ & $\mathrm{R} / \mathrm{R}$ & - & - & - & - & - & - & - & - \\
\hline 15 & Female-20 & $\mathrm{MC}$ & $\mathrm{R} / \mathrm{R}$ & - & - & - & - & + & + & + & + \\
\hline 16 & Male-19 & NS & $\mathrm{R} / \mathrm{R}$ & - & - & - & - & - & - & - & - \\
\hline 17 & Male-49 & NS & $\mathrm{R} / \mathrm{R}$ & - & - & + & + & - & - & + & + \\
\hline
\end{tabular}

Practice changing developments have been reported in solid tumors, mostly in melanoma. On the other hand there are successfull results in hemopoietic neoplasias and this field will open new avenues in these disorders in near future. The most important results among hemopoietic neoplasias have been shown in cases with R/R HL. Cases with HL treated by $4-5$ lines of therapies including brentuximab vedotin and stem cell transplantation have been included in two studies. Response rates in this unlucky group of patients were stunning. In phase I study covering 23 cases with R/R HL, ORR was $87 \%$ (CR and PR rates were 17 and $70 \%$ respectively). Tumor analysis from 10 patients confirmed increased expression of PD-L1 and PD-L2 in all [15].

Keynote-013 study presented at ASH 2014 meeting is a phase Ib study covering 31 cases treated by pembrolizumab, in this study ORR was $66 \%$ with $21 \% \mathrm{CR}$ rate. PD-L1 expression was detected in $100 \%$ of evaluable samples (16). These results suggest that CBT is useful in cases with $\mathrm{R} / \mathrm{R}$ HL and $\mathrm{PD}-1 / \mathrm{PD}-\mathrm{L} 1$ expression may be a predictive markers, at least, in some of these cases. However there is no data in these cases which samples: in first diagnosis or relapsed samples have been evaluated for PD-1/PD-L1 expression.

With the development of these immunotherapies, much interest and energy has been focused on to find a useful predictive marker. However this is not a simple question to be easily answered. There are many questions and problems at this point. Questions are: 1-Which PD-1 or PD-L1 MoAb most accurately determines PD-1 or PD-L1 expression, 2-Which cut off should be utilized to determine the PD-1/ PD-L1 positivity: in many clinical trials $5 \%$ expression in the tumor epithelium has been defined. However this cut off is arbitrary and results are variable. At this point, some studies have defined the cutoff ranging from $1 \%$ to $50 \%$ and in some other studies this threshold has been defined as low (1-5\%), moderate (5-10\%) and high (>10\%) expression. In some of these studies it was found a correlation between higher expression and better response. However it has been shown many times that in some tumors does not respond despite the presence of PD-L1 expression and some respond to CBT despite the absence of PD-L1 expression, 3-We do not know which additional components have a role in predicting response: Tumor infiltrating lymphocytes, PD-L2 expression, mismatch-repair deficiency etc, 4-Where we should measure PD-L1 protein: in tumor cells, stroma (immune infiltrating cells) or in both, 5 -Which method is more reproducible to detect the PD-L1? Quantitative immunofluoroscence, RNA based method or standard immunohistochemistry, 6-Expression pattern of PD-L1 which may be cytoplasmic, membranous or none and this staining pattern may be important. It seems that membranous staining may be predicitive for response to therapy [2,14], 7-Importantly the characterization of PD-1 expression is more difficult in hemopoietic neoplasias, especially in HL due to polymorphic infiltration, compared with solid tumors and we need more standardized measures for these cases. When we looked the available data, results are very chaotic. Preliminary results in 2012 suggested a relationship between PD-L1 expression on tumor cells and objective response rate. According to the PD-L1 expression, 9 of 25 patients with PD-L1 expression showed objective response (36\%) while none of the 17 patients without PD-L1 expression showed response [2]. After this information many studies explored the potential biomarkers predicting response to $\mathrm{PD}-1$ pathway treatment $[2,14,16-19]$. Although in some series it has been found close correlation between the higher response to CBT in cases with stronger PD-L1 expression compared with poor PD-L1 expression, PD-L1 expression on the tumor cell surface may not be the sole determinant of sensitivity to PD-1 blockade. The definition of this result may be due to, as mentioned before, choosing the threshold and MoAb problem in in PD-L1 positivity. Today in spite of huge data from different companies and academic centers for different MoAbs to determine the PD-L1 positivity, we have little data to help choose the best diagnostic MoAb and/or threshold [14,19-22]. Although initial data obtained in solid tumors suggested that PD-L1 expression on tumor cells was the strongest determinant of response to PD-1 blockade, it has been reported meaningful response rate in apperently PD-L1 (-) tumors [5]. In fact, at least in some cases, the tumor microenvironment may determine response more strongly than PD-L1 expression on tumor cells [19]. However complex status is ongoing in melanoma trials and in other tumors due to different stage of the cancers analysed, PD-L1 $\mathrm{MoAb}$ used for IHC staining, laboratory techniques for IHC staining and treatment history [23-25]. False positive and negative results must be considered for the stratification of the patients for CBT. The other important point is the expression of $\mathrm{PD}-\mathrm{L} 1$ on infiltrating lymphocytes, 
monocytes, and macrophages. This status is very important in HL studies due to very high cell infiltration around the RS clls. Therefore the role of PD-L1 expression may vary depending on tumor type and therapeutic approaches used in previous periods [26,27]. PD-1 pathway is engaged by many different hemopoietic neoplasias and that PD-1 ligand expression by tumor cells or in microenvironment of the correlates with more aggressive or refractory disease [5]. PDL1 expression has been found to be higher in high risk and more refractory cases with myelodysplastic syndrome [27]. Additionally it has ben shown that PD-L1 expression is enhanced by treatment with hypomethylating agents. This finding suggests that PD-L1 expression may be associated with more aggressive disease behavior and treatment resistance and CBT can be therapeutically useful in this disease $[28,29]$. The increased expression of programmed death pathway markers after anti-neoplastic treatments in our study may suggest the more aggressive biology and no response to conventional chemotherapeutic agents. The timing of anti-PD-1 therapy plays a crucial role in maximizing therapeutic benefit. The use of anti-PD-1 therapy before, after or concurrently with chemotherapy will be changed the responses dramatically. For this reason timing of immunotherapy is very critical for the beneficial effects of CBT in addition to the expression PD-1 and its ligands in tumors and/or its microenvironment [26].

We found increased expressions of PD-1 and/or PD-L1 after treatment in the majority of cases with PR HL and R/R HL. Expressions were detected both in ME and in RS cells. Although a relevant expression ("more than 5\%") of PD-1 in the ME and on H/RS was documented in more patients post-treatment than prior treatment (8 vs 3 and 4 vs 0 patients) and more patients showed a relevant expression of PD1-L1 after treatment than prior treatment (9 vs 3 pts with regard to the ME and 5 vs 2 pts with regard to the H/RS cells), the increased expression of PD-1 and PD-L1 cannot be proven for the majority of patients. Furthermore, an increased expression of PD-1 and PD-1L1 post-treatment was particulary shown for the ME and to a lesser extent on H/RS cells. In contrast to prior studies $[5,15]$ which documented a relevant PD-L1 expression in H/RS-cells prior treatment with the anti-PD1 antibody in nearly all examined patients, our analysis showed PD-L1 expression in H/RS-cells in 5 of 17 samples and in the ME in 9 of 17 samples at relapse. This difference might be caused by the use of different anti-PD-1-L1 antibodies, by the application of different staining scores or other technical problems. Other limitation of our work is the lack of analyses for PD-L2 expression, MMR deficiency and 9p24.1 amplification which are important parameters for the evaluation of PD-1 pathway.

It is very well known that standard treatment is not clear and there is no good choice in cases with PR HL. On the other hand salvage chemotherapy and high dose chemotherapy/stem cell transplantation is standard in R/R HL cases, but this approach is beneficial in only a limited number of these cases. Brentuximab vedotin is an important molecule in these cases as a bridge for transplantation. In a similar way successfull results have been reported with checkpoint inhibitors in cases with R/R HL. Here we showed that PD-1/PD-L1 expressions increase in cases with PR and R/R HL. For this reason CBT is important therapeutic oppurtunity in cases with $\mathrm{PR}$ and R/R HL cases. Increased expressions of PD1 and/or PD-L1 in RS cells and/or ME may be important in the management of these cases. If PD-1/PD-L1 will be a stratification factor in deciding CBT, it will be ueseful to determine/ check the expressions of programmed death pathway biomarkers in relapse tissue samples.

In summary the most critical point is to understand the most powerful strategy to detect which patients are more likely to benefit from anti-PD-1/PD-L1 blockade and potential biomarkers for improved patient care and also to prevent cost toxicity. In recent times it has been proposed that predictive value of PD-L1 expression may be important to decide the therapy with single checkpoint inhibitor or their combination in melanoma [14]. By analogy this may be which may be a guide for the management of R/R HL. PD-1 and PD-L1 are dynamic merkers changing over time as a result of evolving immune responses, chemo and/or radiation may contribute to these changes. So the detection of PD-1 and/or PD-L1 expressions in samples taken during relapse may be more informative and useful in the CBT with PD-1/PD-L1 inhibitors.

\section{Conflict of interest}

The authors report no conflict of interest

\section{Ethical approval}

This study has been approved by Cukurova University Medical Faculty Ethical comitte for non-invasive research.

\section{Funding}

This study has been supported by Cukurova University Research Fund.

\section{References}

1. Postow MA, Callahan MK, Wolchok JD (2015) Immune Checkpoint Blockade in Cancer Therapy. J Clin Oncol 33: 1974-1982. [Crossref]

2. Topalian SL, Hodi FS, Brahmer JR, Gettinger SN, Smith DC, et al. (2012) Safety, activity, and immune correlates of anti-PD-1 antibody in cancer. $N$ Engl J Med 366: 2443-2454. [Crossref]

3. Robert C, Ribas A, Wolchok JD, Hodi FS, Hamid O, et al. (2014) Anti-programmeddeath-receptor-1 treatment with pembrolizumab in ipilimumab-refractory advanced melanoma: a randomised dose comparison cohort of a phase 1 trial. Lancet 384: 11091117.

4. Bracarda S, Altavilla A, Hamzaj A, Sisani M, Marrocolo F, et al. (2015) Immunologic checkpoints blockade in renal cell, prostate, and urothelial malignancies. Semin Oncol 42: 495-505.

5. Armand P (2015) Immune checkpoint blockade in hematologic malignancies. Blood 125: 3393-3400.

6. Kater AP, van der Windt GJ (2015) PD-L1 blockade: rejuvenating T cells in CLL. Blood 126: 126-128. [Crossref]

7. Eyre TA, Collins GP (2015) Immune checkpoint inhibition in lymphoid disease. $\mathrm{Br} J$ Haematol 170: 291-304. [Crossref]

8. Green MR, Monti S, Rodig SJ, Juszczynski P, Currie T, et al. (2010) Integrative analysis reveals selective 9p24.1 amplification, increased PD-1 ligand expression, and further induction via JAK2 in nodular sclerosing Hodgkin lymphoma and primary mediastinal large B-cell lymphoma. Blood 116: 3268-3277.

9. Yamamoto R, Nishikori M, Kitawaki T, Sakai T, Hishizawa M, et al. (2008) PD-1PD-1 ligand interaction contributes to immunosuppressive microenvironment of Hodgkin lymphoma. Blood 111: 3220-3224.

10. Green MR, Rodig S, Juszcynski P, Ouyang J, Sinha P, et al. (2012) Constitutive AP-1 activity and EBV infection induce PD-L1 in Hodgkin lymphomas and posttransplant lymphoproliferative disorders: implications for targeted therapy. Clin Cancer Res 18: $1611-1618$

11. Paydas S, BaÄŸ̈̈ $\pm r$ E, Seydaoglu G, Ercolak V, Ergin M (2015) Programmed death-1 (PD-1), programmed death-ligand 1 (PD-L1), and EBV-encoded RNA (EBER) expression in Hodgkin lymphoma. Ann Hematol 94: 1545-1552. [Crossref]

12. Chen BJ, Chapuy B, Ouyang J, Sun HH, Roemer MG, et al. (2013) PD-L1 expression is characteristic of a subset of aggressive B-cell lymphomas and virus-associated malignancies. Clin Cancer Res 19: 3462-3473. [Crossref]

13. Muenst S, Hoeller S, Dirnhofer S, Tzankov A (2009) Increased programmed death-1+ tumor infiltrating lymphocytes in classical Hodgkin lymphoma substantiate reduced overall survival. Hum Pathol 40: 1715-1722. 
14. Mahoney KM, Freeman GJ, McDermott DF (2015) The Next Immune-Checkpoint Inhibitors: PD-1/PD-L1 Blockade in Melanoma. Clin Ther 37: 764-782. [Crossref]

15. Ansell SM, Lesokhin AM, Borrello I, Halwani A, Scott EC, et al. (2015) PD-1 blockade with nivolumab in relapsed or refractory Hodgkin's lymphoma. $N$ Engl $J$ Med 372: 311-319.

16. Moskowitz A, Ribrag V, Michot JM(2014) PD-1 blockade with the monoclonal antibody pembrolizumab in patients with classical Hodgkin lymphoma after brentuximab vedotin failure: preliminary results from a phase Ib study (KEYNOTE-013). 56th ASH Annual Meeting, San Francisco, Calif.

17. Thompson RH, Kuntz SM, Leibovich BC (2006) Tumor B7-H1 is associated with poor prognosis in renal cell carcinoma patients with long-term follow up. Cancer Res 66: 3381-3385.

18. Srivasta N, McDermott D (2014) Update on benefit of immunotherapy and targeted therapy in melanoma: the changing landscape. Cancer Manag Res 6: 279-289.

19. Herbst RS, Soria JC, Kowanetz M, Fine GD, Hamid O, et al. (2014) Predictive correlates of response to the anti-PD-L1 antibody MPDL3280A in cancer patients. Nature 515: 563-567

20. Powles T, Eder JP, Fine GD, Braiteh FS, Loriot Y, et al. (2014) MPDL3280A (antiPD-L1) treatment leads to clinical activity in metastatic bladder cancer. Nature 515: 558-562. [Crossref]
21. Taube JM, Klein A, Brahmer JR, Xu H, Pan X, et al. (2014) Association of PD-1, PD-1 ligands, and other features of the tumor immune microenvironment with response to anti-PD-1 therapy. Clin Cancer Res 20: 5064-5074. [Crossref]

22. Garon EB, Rizvi NA, Hui R, Leighl N, Balmanoukian AS, et al. (2015) Pembrolizumab for treatment of non-small-cell lung cancer. N Engl J Med 372: 2018-2128.

23. Robert C, Long GV, Brady B, Dutriaux C, Maio M, et al. (2015) Nivolumab in previously untreated melanoma without BRAF mutation. $N$ Engl J Med 372: 320-330. [Crossref]

24. Moreno BH, Ribas A. Anti-programmed cell death protein-1/ligand-1 therapy in different cancers. Br J Cancer 112: 1421-1427.

25. Mahoney KM, Atkins MB (2014) Prognostic and predictive markers for the new immunotherapies. Oncology (Williston Park) 28 Suppl 3: 39-48. [Crossref]

26. Wolchok JD, Kluger H, Callahan MK, Postow MA, Rizvi NA, et al. (2013) Nivolumab plus ipilimumab in advanced melanoma. $N$ Engl J Med 369: 122-133. [Crossref]

27. Wolchok JD, Kluger H, Callahan MK, Postow MA, Rizvi NA, et al. (2013) Nivolumab plus ipilimumab in advanced melanoma. $N$ Engl J Med 369: 122-133. [Crossref]

28. Kondo A, Yamashita T, Tamura H, Zhao W, Tsuji T, et al. (2010) Interferon-gamma and tumor necrosis factor-alpha induce an immunoinhibitory molecule, B7-H1, via nucelar factor-kappaB activation in blasts myeloblastic syndromes. Blood 116: 1124-1131.

29. Bryan LJ, Gordon LI (2015) Releasing the Brake on the Immune System: The PD-1 Strategy for Hematologic Malignancies. Oncology (Williston Park) 29: 431-439. [Crossref]

Copyright: @2018 Paydas S. This is an open-access article distributed under the terms of the Creative Commons Attribution License, which permits unrestricted use, distribution, and reproduction in any medium, provided the original author and source are credited. 